\title{
Diagnostic Performance of CT Compared to PET/CT in Evaluating Bone Metastasis: An Initial Experience from Nepal
}

\author{
Rajive Raj Shahi, ${ }^{1}$ Sampanna Jung Rayamajhi, ${ }^{2}$ Sagar Maharjan, ${ }^{2}$ Sujata Panta ${ }^{2}$ \\ 'Department of Radiology, Nepali Army Institute of Health Sciences, Kathmandu, Nepal ${ }^{2}$ Kundalini Diagnostic Center, \\ Chandol, Kathmandu, Nepal
}

\section{ABSTRACT}

\section{Introduction}

Bone metastasis is frequently encountered in different cancers. Computed tomography (CT) is commonly used to detect bone metastasis. Positron emission tomography (PET) has only recently been introduced in Nepal. Various studies have shown PET/CT to be superior to CT alone in identifying bone metastasis. This study aims to compare the utility of CT scan compared to F-18 FDG PET/CT for detecting bone metastasis in common non-hematological malignancies.

\section{Methods}

The medical records of cancer patients referred to us between from September 2019 to April 2020 were reviewed retrospectively. Two radiologists and two nuclear medicine physicians read the CT and PET/CT scans respectively, performed as part of the same study. Results were categorized as positive, negative of equivocal. The sensitivity, specificity and accuracy of CT alone was evaluated compared to PET/CT.

\section{Results}

A total of 365 patients were included in the study. Out of 365 patients, bone metastasis was detected in 53 patients $(14.5 \%)$. CT alone was true positive in 40 patients, true negative in 311 patients, false positive in 1 patient and false negative in 13 patients. (5 equivocal cases considered as negative). On per-patient analysis, CT alone when compared to PET/CT had sensitivity, specificity, and overall accuracy of $75.4 \%, 99.6 \%$ and $96.1 \%$ respectively for detection of bone metastasis.

\section{Conclusions}

Our study highlights that metabolic alteration in bone metastasis are identified by F-18 FDG PET/CT even before morphological changes are apparent on CT. Further, F-18 FDG PET/CT is extremely helpful if CT findings are equivocal for bone metastasis.

Keywords: bone metastasis; F-18 FDG PET/CT; Fluorodeoxyglucose F18. 


\section{INTRODUCTION}

Cancers of the breast, lung, GI, sarcomas, head and neck etc. are common in Nepal and bone metastasis is frequently encountered in them. Conventional imaging modalities such as plain radiographs and $\mathrm{CT}$ are frequently used to evaluate bone metastasis. F-18 FDG PET/CT which is already an indispensable tool in the field of oncology was only recently introduced in Nepal. Studies have shown that using CT alone in cancer patients may miss bone metastasis which may inaccurately downstage a patient and lead to suboptimal cancer management. In our study we compare performance of CT compared to PET/CT to evaluate bone metastasis in a wide range of malignancies with the aim of evaluating how CT is performing compared to PET/CT in detecting bone metastasis and whether PET/CT is a problem solver whenever CT is in doubt/ equivocal over the nature of the bone lesion.

\section{METHODS}

The medical records of cancer patients (except hematological malignancies) referred to us for staging, restaging, response evaluation or suspected recurrence, between from September 2019 to April 2020 were reviewed retrospectively. The study was approved by National Health and Research Council IRB. F-18 FDG-PET/CT imaging was acquired utilizing an integrated PET/CT device (Philips Ingenuity TF PET/CT). The integrated CT system is a 128 multi-slice scanner. The acquisition of co-registered CT and PET images was performed in one session. Patients fasted for at least 4 hours before the scan and blood glucose level was ensured to be $<200 \mathrm{mg} / \mathrm{dl}$ before FDG injection. Images were acquired $45-60 \mathrm{~min}$ post intravenous administration of $(0.14 \mathrm{mCi} / \mathrm{kg})$ of $\mathrm{F}-18$ FDG. IV contrast administration (80-120 mL) of a low-osmolarity iodinated contrast agent was used. PET scan was performed in the same position. Attenuation correction was done by unenhanced CT. All CT, and PET/CT images were reconstructed and viewed on a Philips workstation, which provided multi-planar reformatted PET, CT, and fused PET/CT images with linked cursors as well as 3D maximum intensity projection (MIP). For F-18 FDG PET/ CT evaluation, two nuclear medicine physicians who had at-least 7 years of experience in nuclear medicine, had no clinical information regarding the presence or absence of bone metastasis evaluated. Physicians analyzed the images independently but reached consensus for the final diagnosis. The site and intensity of FDG uptake (standardized uptake values; SUVmax) was noted. Presence of visually significantly increased focally increased FDG uptake apparent within the bone was considered metastatic. The absence of uptake was considered as a negative. Faint FDG uptake similar to the bone marrow was considered equivocal. Two radiologists with at-least 7 years of experience in CT, evaluated the contrast enhanced CT only data of patients from the same PET/CT study without having access to the PET images. A lesion was considered a positive finding on CT (malignant) if it appeared ill-defined, irregular, or heterogeneously blastic, lytic with irregular sclerotic margins, associated with cortical destruction or extra osseous soft tissue component. Bone lesions were considered benign if they were well defined, homogenously, and fully sclerotic, and lytic with regular sclerotic margins particularly when localized to the vertebral end plates or articular surfaces. Lesions were characterized into benign, malignant, or equivocal after consensus between the two radiologists. Morphology of the lesion (sclerotic, osteolytic, mixed, presence of soft tissue) was noted. Radiologists were blinded to findings on PET. Reporting by nuclear medicine physician and radiologist were independent of each other. 


\section{RESULTS}

The study included 365 patients with nonhematological malignancies. Out of 365 patients bone metastases was detected in 53 patients $(14.5 \%)$. Their age range is $10-81$ years; with mean age: 53.52 years \pm 14.32(SD). The clinicopathological characteristics of these patients are detailed in Table 1.

Table 1.

\begin{tabular}{|l|c|}
\hline Parameters & Value \\
\hline Age (years) & $53.52 \pm$ \\
Mean & 14.32 \\
Range & $10-81$ \\
\hline Sex (n) & \\
Male & 24 \\
Female & 29 \\
\hline Type of malignancy (n=53) & \\
Breast & 15 \\
Non-small cell lung cancer (NSCLC) & 14 \\
Prostate & 2 \\
GI tract & Esophagus \\
& $(1)$, stomach \\
Ovary & $(2)$, colon (2) \\
Head and neck & 1 \\
Unknown primary & 3 \\
Sarcoma & 1 \\
Melanoma & 4 \\
NET (grade III) & 3 \\
Urinary bladder & 3 \\
Renal & 1 \\
\hline Type of morphological change seen on CT & \\
Blastic metastasis & 16 \\
Lytic metastasis & \\
Mixed & \\
Equivocal CT change (lytic/blastic) & \\
No CT changes & \\
Lesions with soft tissue component (lytic/blastic) & 6 \\
\hline
\end{tabular}

Both F-18 FDG PET/CT and CT alone detected bone metastasis (Figure 1 and 2). F-18 FDG PET/CT detected bone metastasis in 53 patients and 312 patients did not have bone metastasis. No equivocal results were seen with PET/CT.

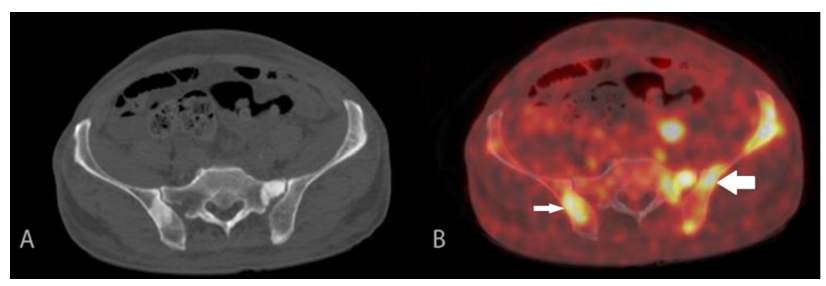

Figure 1. 81-year- old male with prostate adenocarcinoma, status post prostatectomy. Axial CT (image A) demonstrating sclerotic bone metastases. Axial PET/CT image (image B) demonstrating increased FDG uptake in the sclerotic regions (thin arrow) as well as in areas without CT change (thick arrow).

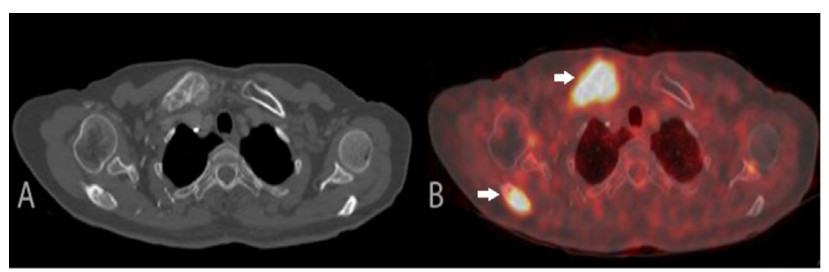

Figure 2. 58-year-old with female with T8 vertebral biopsy compatible with metastatic squamous cell carcinoma. Axial CT (image A) demonstrating lytic bone metastases in the right clavicle and scapula (arrows) with corresponding increased FDG uptake in the PET/CT image (image B).

CT alone was true positive (TP) in 40 patients, true negative (TN) in 311 patients, false positive (FP) in 1 patient and false negative (FN) in 13 patients. (5 equivocal cases considered as negative). Characteristics of bone metastasis detected on these two techniques are demonstrated in Table 1. On per-patient analysis, CT alone when compared to PET/CT had sensitivity, specificity, and overall accuracy of $75.4 \%, 99.6 \%$ and $96.1 \%$ 
respectively for detection of bone metastasis.

Out of the 53 patients who had bone metastasis, CT alone was unable to detect any bone lesion in 8 patients $(15 \%)$ as shown in (Figure 3 and 4). These patients $(n=8)$ had clear evidence of bone metastasis in F-18 FDG PET/CT. Further in this subgroup, 7 patients had clear evidence of FDG avid extraosseous metastases and 6 of them had multiple FDG avid bone lesions. Characteristics of these patients are demonstrated in Table 2.
E). Corresponding PET/CT images (image B, D, F) with hypermetabolic lesions in the vertebrae and right acetabulum (arrows) suggesting metastasis.

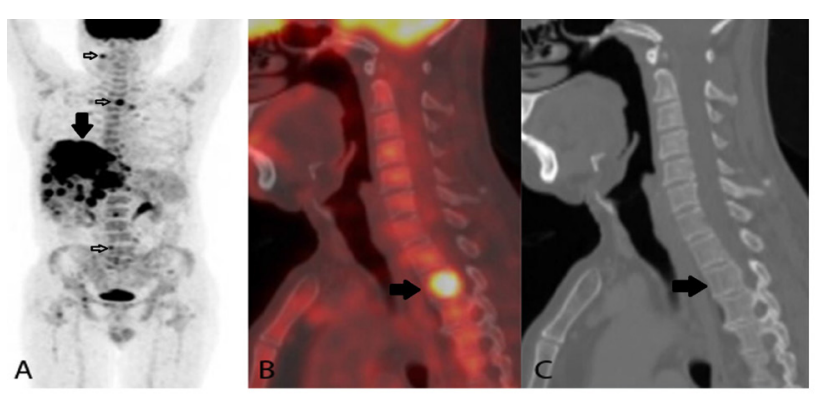

Table 2. Characteristics of F-18 FDG PET/CT positive and CT negative cases

\begin{tabular}{|l|l|l|l|l|}
\hline SN & Primary tumor & Site of bone metastasis & $\begin{array}{l}\text { SUVmax of the } \\
\text { most avid lesion }\end{array}$ & Sites of visceral metastasis \\
\hline 1 & NSCLC & vertebrae & 11.2 & Multiple liver \\
\hline 2 & Choroidal Melanoma & Vertebrae, ribs, pelvis, femur & 9.5 & $\begin{array}{l}\text { Multiple liver, abdominal } \\
\text { lymph nodes }\end{array}$ \\
\hline 3 & Breast & Vertebrae, pelvis & 6.4 & $\begin{array}{l}\text { Multiple liver, abdominal and } \\
\text { mediastinal nodes }\end{array}$ \\
\hline 4 & Colon & Vertebrae, ribs, pelvis, femur & 7.4 & $\begin{array}{l}\text { Multiple liver, peritoneal and } \\
\text { muscle }\end{array}$ \\
\hline 5 & Ovary & Right humerus & 3.6 & $\begin{array}{l}\text { Multiple } \\
\text { mesenteric }\end{array}$ \\
\hline 6 & Sarcoma femur & Sacrum, vertebra & 4.8 & bones only \\
\hline 7 & Sinonasal Melanoma & femur & 4.6 & Multiple lungs and liver \\
\hline 8 & $\begin{array}{l}\text { Grade III NET pancreas } \\
\text { Ki 67:50-60\% }\end{array}$ & Vertebrae and pelvis & 6.0 & Multiple liver \\
\hline
\end{tabular}

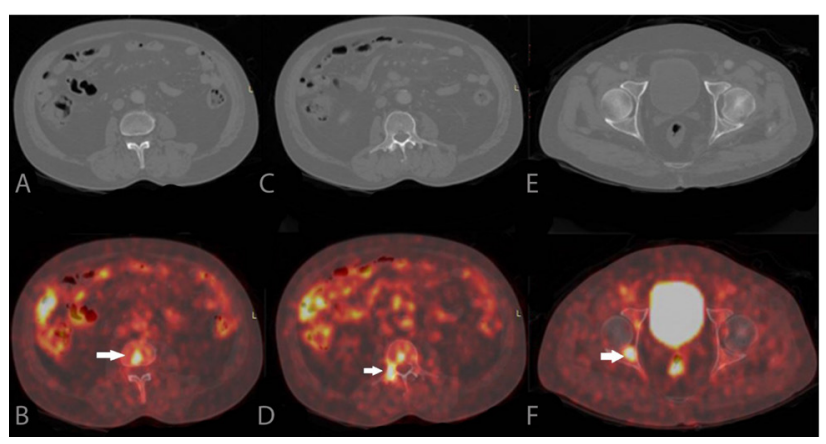

Figure 3. 76-year-old male with neuroendocrine tumor of the pancreas (grade III). Axial CT images of the spine and pelvis with no demonstrable bone metastases (images A, C and
Figure 4. 64-year-old male with right eye choroidal melanoma. Maximum Intensity projection images of PET/CT (image A) demonstrating multiple bone metastasis (small arrows) and multiple liver metastasis (thick solid black arrow). PET/CT (image B) demonstrating the thoracic vertebra metastasis (arrow) which is not seen on CT (image C).

In 5 patients (9.4\%) CT demonstrated equivocal findings (Figure 5). In these cases, F-18 FDG PET/CT showed increased focal FDG uptake 
at these sites and were highly suspicious for metastasis in these patients. Further in some of these patients there were high focal FDG uptake at other osseous sites where CT did not demonstrate any morphological change.
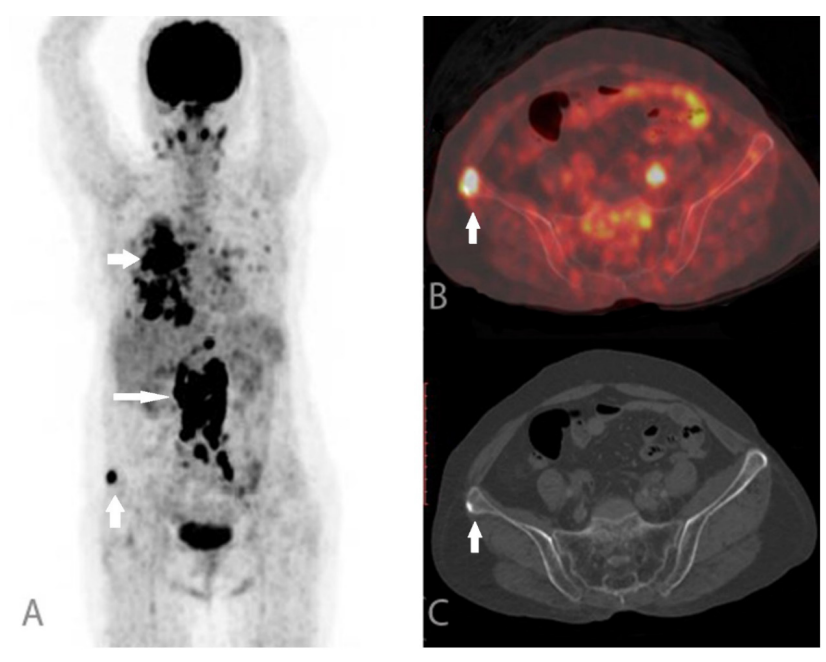

Figure 5. 71-year-old female with right lung malignancy. Maximum Intensity projection images of PET/CT (image A) demonstrating primary malignancy in the right lung (small horizontal arrow), metastatic abdominal lymph nodes (long horizontal arrow) and bone metastasis (vertical arrow). PET/CT (image B) demonstrating the right iliac bone metastasis (arrow) which is seen as small equivocal sclerotic lesion on CT (image C).

In 1 patient with breast cancer on follow up after treatment, CT demonstrated multiple dense sclerotic lesions. This patient had extraosseous metastasis, but the bone lesions were non FDG avid and were stable since prior imaging, indicating healed osseous metastases and hence were falsely positive on CT (Figure 6). In a subgroup of 11 patients CT demonstrated morphological changes of bone metastasis in at least one bone (either lytic or sclerotic) but in these patients F-18 FDG PET/CT showed additional multiple sites of bone metastasis which had no changes on CT. The primary tumor site in these patients were lung $(n=2)$, breast $(n=3)$, prostate $(n=1)$, urinary bladder $(n=1)$, colon $(n=1)$, tongue $(\mathrm{n}=1)$, stomach $(\mathrm{n}=1)$ and rhabdomyosarcoma $(\mathrm{n}=1)$.

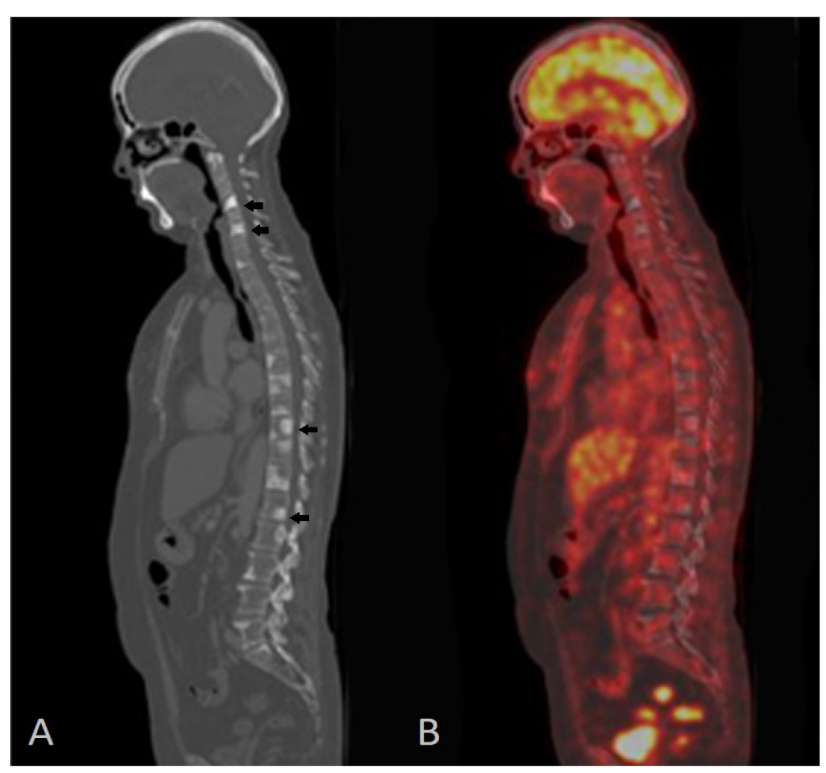

Figure 6. 61-year-old female with breast cancer post chemotherapy. Sagittal CT (image A) demonstrating multiple sclerotic lesions in the vertebrae (arrows). Sagittal PET/CT (image B) does not show increased FDG uptake in them, compatible with treated metastases.

One patient with prior systemic therapy there was diffuse sclerotic bone lesions on CT. However, only one lesion was metabolically active on PET indicating the viability of metastasis whereas all other were without metabolic activity on PET indicating treated metastasis (Figure 7).

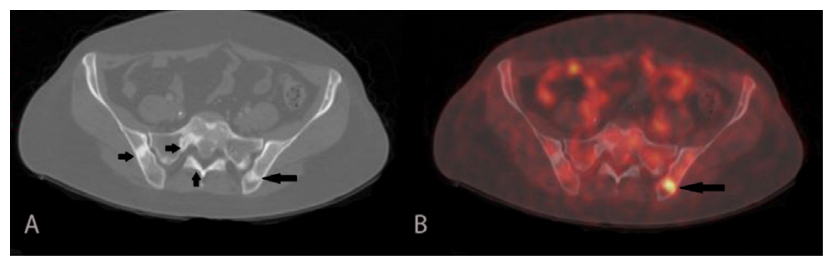

Figure 7. 44-year-old female with history of metastatic breast cancer, post chemotherapy and radiation, currently on Tamoxifen. Axial CT (image A) demonstrating multiple sclerotic lesions (arrows) in the pelvic bones. Axial PET/ CT (image B) demonstrating only one lesion to be metabolically active metastasis (arrow) 
and others do not demonstrate hypermetabolic compatible with treated disease.

\section{DISCUSSION}

Cancers of the breast, lung, prostate are common worldwide, South Asia including Nepal. ${ }^{1,2}$ Bone is one of the most common sites of metastasis from common solid tumors. ${ }^{3}$ Identifying bone metastasis is of paramount importance as it may have a significant impact in patient management. For detecting bone metastasis, oncologists here have typically relied on conventional imaging modalities, mainly CT scans. F-18 FDG PET/ $\mathrm{CT}$ is a robust and reliable diagnostic tool for evaluating various malignancy. Although it is not a new imaging modality, it was only recently introduced in Nepal.

In this study we aimed to evaluate the performance of CT compared to F-18 FDG PET/ CT in identifying bone metastasis in patients with different common non hematological malignancies referred to us from many hospitals all over Nepal.

Both F-18 FDG PET/CT and CT alone successfully detected metastatic bone disease in our patients. In our study there werelytic, blastic, mixed lesions and few osseous lesions also demonstrated soft tissue component. F-18 FDG PET/CT showed abnormal high metabolic activity in both lytic and blastic lesions. From our early experience, it is evident that F-18 FDG PET/CT is clearly more superior to CT in identifying bone metastasis. Similar findings were shown by prior studies. ${ }^{4.5}$ In patient-based analysis, in 8 of our patients, CT demonstrated no morphological changes to suggest bone metastasis whereas F-18 FDG was positive, clearly indicating that $\mathrm{CT}$ alone may miss early metastatic lesions, similar findings have been described in previous studies. ${ }^{6-10}$ Caglar et al in their study of patients with breast cancer, also demonstrated that findings of PET positive and CT negative metastatic lesions were not uncommon. ${ }^{11}$ Although we did not analyze the impact in patient management, identification of such lesions may have possibly made a difference in selecting the most appropriate line of intervention.

In 5 patients where CT was equivocal, F-18 FDG PET/CT clearly identified osseous metastasis. These patients did not show clear morphological changes of metastasis on CT alone and some were of questionable significance. This highlights the fact that F-18 FDG PET/CT is helpful in identifying osseous metastatic lesions when CT findings are ambiguous.

In 12 patients who had clear morphological changes of bone metastasis in at least one site, F-18 FDG PET/CT showed additional sites of bone involvement where CT did not show any morphological change, highlighting the fact that CT alone may underestimate the true extent of osseous metastasis in a patient. Identification of such additional sites may change patient management, for example change the radiation field of view, may limit surgical options etc.

Yamaguchi et al. in their study showed that osseous metastatic lesions are frequently intertrabecular where the trabecular bone structure is retained and hence no osteolytic or osteoblastic changes. ${ }^{12} 8$ patients where CT was entirely negative and 12 patients where many extra additional (CT negative leisions) likely had intertrabecular metastasis which was effectively picked up on F-18 FDG PET/CT. Various cases have been reported in literature where patients had widespread bone metastasis effectively picked up by F-18 PDG PET/CT but being intertrabecular in nature, no $\mathrm{CT}$ changes was seen. ${ }^{13-14}$

F-18 FDG PET provides metabolic information of a tumor which helps to assess treatment response. A metastatic bone lesion may initially demonstrate high FDG uptake but following 
treatment such as chemotherapy or radiotherapy the initial high uptake may decrease in intensity or resolve indicating treatment response. ${ }^{15-16}$ One of our patients with prior systemic therapy there was diffuse sclerotic bone lesions. However, only one lesion was metabolically active on PET indicating the viability of metastasis whereas all other were without metabolic activity on PET indicating treated metastasis. Thus, CT alone was of limited use in this patient as it could not identity whether the sclerotic lesions were active metastasis or treated, hence CT alone would have falsely overestimated the osseous metastatic burden. Further this patient demonstrated bone only metastasis and had no visceral disease.

There are certain limitations of our study. This study is limited by its retrospective design. Majority of our patients had NSCLC and inflammatory ductal carcinoma of the breast which are known to demonstrate high
FDG uptake. Our patient cohort had only few malignancies such as mucinous cancers of the gastrointestinal tract, prostate cancer and renal cell cancers which are known for low FDG uptake. This may have resulted in selection bias. Since our center receives scan referrals from different hospitals all over the country, 18 F-FDG PET/CT results and histologic subtype/ nuclear grade could not be compared.

\section{CONCLUSIONS}

Our study highlights that metabolic alteration in bone metastasis are identified by F-18 FDG PET/CT even before morphological changes are apparent on CT. Thus F-18 FDG PET/CT is more useful than CT in identifying bone lesions early which may have significant impact in patient management. Further, F-18 FDG PET/CT is also extremely helpful if findings which are equivocal for bone metastasis on CT.

SM. Diagnosis of bone metastases: a meta-analysis comparing ${ }^{18} \mathrm{FDG}$ PET, CT, MRI and bone scintigraphy. Eur Radiol. 2011;21(12):2604-17. PMID: 21887484 .

5. Talbot JN, Paycha F, Balogova S. Diagnosis of bone metastasis: recent comparative studies of imaging modalities. Q J Nucl Med Mol Imaging. 2011;55(4):374-410. PMID: 21738113.

6. Evangelista L, Panunzio A, Polverosi R, Ferretti A, Chondrogiannis S, Pomerri $\mathrm{F}$, et al. Early bone marrow metastasis detection: the additional value of FDG-PET/CT vs. CT imaging. Biomed Pharmacother. 2012;66(6):448-53. PMID: 22902054.

7. Nakamoto Y, Cohade C, Tatsumi M, Hammoud D, Wahl RL. CT appearance of bone metastases detected with 
FDG PET as part of the same PET/CT examination. Radiology. 2005;237(2):62734. PMID: 16244271.

8. Ahmed F, Muzaffar R, Fernandes H, Tu Y, Albalooshi B, Osman MM. Skeletal Metastasis as Detected by 18F-FDG PET with Negative CT of the PET/CT: Frequency and Impact on Cancer Staging and/or Management. Front Oncol. 2016;(6):208. PMID: 27777898.

9. Kandathil A, Kay FU, Butt YM, Wachsmann JW, Subramaniam RM. Role of FDG PET/CT in the Eighth Edition of TNM Staging of Non-Small Cell Lung Cancer. Radiographics. 2018;38(7):213449. PMID: 30422775.

10. Wafaie A, Kassem H, Kotb M, Zeitoun R, Ismails. Evaluation of the efficiency of FDG PET/CT in detection and characterization of skeletal metastases. The Egyptian Journal of Radiology and Nuclear Medicine. 2014;45(1):181-90. https://doi. org/10.1016/j.ejrnm.2013.11.007.

11. Caglar M, Kupik O, Karabulut E, HoilundCarlsen PF. Detection of bone metastases in breast cancer patients in the PET/CT era: Do we still need the bone scan? Rev Esp Med Nucl Imagen Mol. 2016;35(1):311. PMID: 26514321.

12. Yamaguchi T, Tamai K, Yamato M, Honma K, Ueda Y, Saotome K. Intertrabecular pattern of tumors metastatic to bone. Cancer. 1996;78(7):1388-94. PMID: 8839543

13. Morita S, Suda T, Oda C, Kobayashi M, Hoshi T, Kanefuji $\mathrm{T}$, et al. The Value of (18)F-FDG PET in the Diagnosis of Intertrabecular Vertebral Metastasis in a Small Cell Lung Cancer Patient with a High Serum CEA Level. Intern Med. 2019;58(3):415-8. PMID: 30146594.

14. Satoh Y, Koizumi K, Nambu A, Araki T. A Case of Massive Bone Metastases From Lung Cancer Detected Only by 18F-FDG PET/CT. Clin Nucl Med. 2015;40(8):e4114. PMID: 26018679

15. Israel $\mathrm{O}$, Goldberg $\mathrm{A}$, Nachtigal $\mathrm{A}$, Militianu D, Bar-Shalom R, Keidar Z, et al. FDG-PET and CT patterns of bone metastases and their relationship to previously administered anti-cancer therapy. Eur J Nucl Med Mol Imaging. 2006;33(11):1280-4. PMID: 16791597

16. Du Y, Cullum I, Illidge TM, Ell PJ. Fusion of metabolic function and morphology: sequential [18F]fluorodeoxyglucose positron-emission tomography/ computed tomography studies yield new insights into the natural history of bone metastases in breast cancer. J Clin Oncol. 2007;25(23):3440-7. PMID: 17592153.

Citation: Shahi R, Rayamajhi S, Maharjan S, Panta S. Diagnostic Performance of CT Compared to PET/CT in Evaluating Bone Metastasis - An Initial Experience from Nepal. JCMS Nepal. 2021 ; 17(4); 352-59. 